Courrier du Centre international Blaise Pascal

15 | 1993

Varia

\title{
Pascal en Roumanie (1965-1993)
}

Horia Lazar

\section{OpenEdition}

Journals

Édition électronique

URL : http://journals.openedition.org/ccibp/598

DOI : $10.4000 /$ ccibp.598

ISSN : 2493-7460

\section{Éditeur}

Centre international Blaise Pascal

\section{Édition imprimée}

Date de publication : 27 février 1993

Pagination : 8-10

ISSN : 0249-6674

\section{Référence électronique}

Horia Lazar, «Pascal en Roumanie (1965-1993)», Courrier du Centre international Blaise Pascal [En ligne], 15 | 1993, mis en ligne le 07 janvier 2016, consulté le 14 septembre 2020. URL : http:// journals.openedition.org/ccibp/598

Ce document a été généré automatiquement le 14 septembre 2020.

Centre international Blaise Pascal 


\title{
Pascal en Roumanie (1965-1993)
}

\author{
Horia Lazar
}

1 Dans la dernière trentaine d'années, la présence de Pascal en Roumanie est moins pauvre qu'on ne s'y attendrait. Malgré la pénurie d'informations et de livres, et aussi la censure exercée sur la publication d'écrits non conformes à l'idéologie du régime, universitaires et chercheurs se sont penchés, depuis 1965, sur les textes pascaliens, en ont fait objet d'étude et de réflexion, et ont réussi même, parfois, à faire publier le résultat de leur travail.

2 Sans être considérable, la production pascalienne en Roumanie est néanmoins marquée. En témoignent plusieurs traductions, trois livres écrits dans des perspectives d'analyse très différentes mais donnant, par cela même, un effet de complémentarité bien manifeste, ainsi qu'une bonne dizaine d'études et d'articles, parus soit dans des revues spécialisées, soit dans des synthèses de philosophie, soit enfin en guise de préface aux traductions.

\section{Traductions}

3 Elles couvrent la majeure partie de l'œuvre pascalienne - les textes les plus connus. En 1966 déjà, Elena Vianu donnait dans une anthologie des moralistes français (Montaigne, Pascal, La Rochefoucauld et La Bruyère) des traductions de fragments des Provinciales et des Pensées, et aussi une version intégrale des Trois Discours sur la condition des grands, pour qu'en 1967 George Iancu Ghidu fît paraître la première partie des Pensées selon l'édition Bossut, ainsi que de petits extraits des Provinciales et des œuvres scientifiques de Pascal. Le projet allait se poursuivre et se préciser, dans le sens de la mise à la disposition du public d'un ouvrage pascalien tout entier : la publication de la traduction intégrale des Provinciales, en 1973. On remarquera, dans les traductions de Ghidu, la rigueur et la clarté de la transposition en roumain du discours pascalien, le souci des nuances et le désir de restituer l'exubérance polémique des Provinciales.

4 En 1978, le lecteur roumain pouvait relire une bonne partie des Pensées dans une nouvelle traduction, due I.A. Badea. Si Ghidu avait utilisé, pour la sienne, les éditions Bossut, Havet, Faugère, Brunschvicg et Chevalier, Badea s'en tiendra au texte établi par 
Brunschvicg, en donnant toutefois les concordances avec l'édition Lafuma. Accompagnée de notes et de petits commentaires (comme celle de Ghidu), la traduction de Badea donne des équivalences plus modernes: le rationalisme pascalien s'y lit parfaitement, avec la finesse de ses distinctions, même si, parfois, les versions proposées sont surprenantes.

En 1992 enfin, on a pu lire en roumain la traduction intégrale des Pensées, faite par Ghidu. Au dire du traducteur lui-même, le texte, tel qu'il se présente dans cette édition, avait été remis à l'éditeur dès 1967, mais il avait été amputé de la seconde moitié par la censure. Il aura donc fallu attendre 25 ans - et le grand bouleversement politique de 1989 - pour qu'il puisse paraitre intégralement.

6 Le lecteur roumain est donc en possession, dorénavant, d'une version intégrale des Provinciales, et aussi des Pensées. On regrette néanmoins l'absence d'une traduction intégrale des Pensées qui suivent le travail de restitution toujours plus rigoureuse des dossiers pascaliens dont le fruit ont été les trois éditions aujourd'hui de référence: Lafuma $(1951,1963)$, Le Guern (1977) et Sellier $(1976,1991)$.

\section{Livres}

7 Un premier Blaise Pascal, celui de Nina Façon, paraissait en 1969. C'est une monographie qui retrace la vie de Pascal et qui, conjointement, suit les étapes de sa formation, en même temps que l'élaboration de ses œuvres. Entre Pascal savant et Pascal apologiste, en passant par Pascal polémiste et moraliste, les gradations et distinctions s'enchaînent, dans une image vivante de l'époque, des milieux intellectuels du XVII siècle et des préoccupations de Pascal lui-même. Le grand mérite du livre est de fournir un tableau animé - et à la fois très nuancé - du mouvement d'idées, des tendances et des conflits idéologiques du siècle classique.

8 Peu après, en 1972, le deuxième Blaise Pascal, dû à Victor Marian et à Maria Popescu, présente les écrits scientifiques du grand Clermontois. Le livre est un aperçu rigoureux des recherches pascaliennes en mathématiques (les coniques, la machine arithmétique, la règle des partis, le triangle arithmétique, la roulette) et en physique (le problème du vide, l'équilibre des liqueurs). Le cheminement des expériences et de la réflexion pascalienne y est décrit avec des références constantes aux conceptions scientifiques de l'époque. La géométrie, les recherches sur la règle des partis et l'hydrostatique de Pascal sont ainsi intégrées dans le débat et les tâtonnements scientifiques de la première moitié $\mathrm{du} \mathrm{XVII}^{\mathrm{e}}$ siècle. La dernière partie du livre est un choix de textes à caractère scientifique : fragments de lettres, de la Préface pour le Traité du Vide, du Traité de l'Équilibre des Liqueurs et du Traité de la Pesanteur de la masse de l'air.

Le dernier Blaise Pascal enfin, paru en 1991, qui est le nôtre, et qui a comme sous-titre Un discours asupra ratiunii (Un discours sur la raison) avec un clin d'œil voulu à Descartes, est issu d'une thèse de doctorat soutenue en 1984 à l'Université de Cluj. Deux idées maîtresses s'y croisent: 1 . celle d'un Pascal rationaliste, «sceptique " (dans le sens étymologique de la skepsis : processus de recherche polémique - Unamuno dit mieux : " agonique »- de la vérité et du sens, opposé à l'assurance dogmatique, qui pose des vérités premières ou institue des principes dont on n'a pas établi - ou éprouvé - le fondement) ; 2. celle du « milieu », qui définit le mieux, croyons-nous, l'état de l'homme pascalien, et dans la source est le paradigme du médiateur: le Christ. Si l'idée d'un Pascal rationaliste est un parti pris méthodologique, solidaire du refus du 
«mysticisme " pascalien ou d'un prétendu caractère "dialectique " (à la manière de Hegel : conflit de tendances dépassé dans la synthèse) de sa démarche, la prééminence du thème du milieu définit, selon nous, les grands traits de l'anthropologie pascalienne. L'homme plongé au milieu de l'infini physique ou dans celui de ses désirs, voué à la dérive perpétuelle, est aussi l'homme promis au salut, s'il est capable de retrouver la bonne vue des choses. Or, l'aptitude à tout comprendre, par-delà l'extrémisme des systèmes philosophiques et la partialité des enjeux de pouvoir, qui est celle-là même du cœur, est réservée au " point haut " d'amour et d'humilité désigné par la passion du Christ. Le corollaire du refus des extrêmes, qui est à l'œuvre dans la conception du milieu, est l'élévation paradoxale dans la «bassesse » de l'être qui renonce à la sagesse du monde pour la folie de la Croix, et la mise en perspective des apparences selon la logique du cœur.

Nous retrouvons ainsi, à la racine de l'anthropologie pascalienne, le modèle scientifique proposé par la "géométrie du hasard ", qui sous-tend aussi la réflexion sur la règle des partis et sur le pari, si bien analysée par Laurent Thirouin dans Le hasard et les règles.

\section{3. Études et articles}

11 Ils sont répertoriés dans la bibliographie ci-dessous. Les études sont d'étendue très variée et de valeur inégale. On remarquera néanmoins la présence, dans la liste, d'un numéro des Annales de l'Université de Bucarest, sorti lors de la commémoration des 350 ans de la naissance de Pascal. Il renferme 19 articles et communications scientifiques, tous en français : autant d'approches fort différentes - et souvent très intéressantes des textes pascaliens et de la problématique qu'ils ouvrent.

\section{BIBLIOGRAPHIE}

\section{Traductions}

Moralisti francezi. Montaigne. Pascal. La Rochefoucauld. La Bruyère. Texte alese, traduse si comentate de Elena Vianu. Bucuresti, Editura pentru Literatură Universală, 1966.

Blaise Pascal, Scrieri alese. Cugetări. Provinciale. Opere stiintifice. Traducere de George Iancu Ghidu. Studiu introductiv de Ernest Stere. Note de George Iancu Ghidu si Ernest Stere. Bucuresti, Editura Stiintifică, 1967.

Blaise Pascal, Scrisori provinciale. Traducere, notite introductive, note si indici de George Iancu Ghidu. Studii de Ion Brŏescu si Florica Neagoe. Bucuresti, Editura Stiintific $\breve{\alpha}, 1973$.

Blaise Pascal, Cuget $\breve{\alpha r i}$ (texte alese). Traducere, note si comentarii de Ion Alex. Badea. Prefat $\breve{\alpha}$ de Romul Munteanu. Bucuresti, Editura Univers, 1978.

Pascal, Cugetŏri. Traducere de George Iancu Ghidu. Studiu introductiv de Ernest Stere. Note de George Iancu Ghidu si Ernest Stere. Bucuresti, Editura Stiintifică, 1992. 


\section{Livres}

Nina Façon, Blaise Pascal, Bucuresti, Editura pentru Literatură Universală 1969.

Horia Lazŏr, Blaise Pascal. Un discours asupra ratiunii, Bucuresti, Editura Stiintifică 1991.

Victor Marian \& Maria Popescu, Blaise Pascal, Bucuresti, Editura Stiintifică, 1972.

\section{Études et articles}

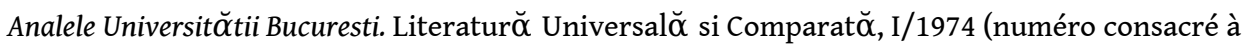
Pascal).

Dan Bŏdŏ̆rŏu, « Amprente pascaliene în filosofia existentei », in Revista de filosofie, 14/ 1967.

Alexandru Boboc, Pascal si axiología contemporană, in Studii de istorie a filosofiei universale, VII, Bucuresti, Editura Academiei, 1979.

Alexandra Emilian, « Points de vue roumains sur Pascal », in Analele Universitŏtii Bucuresti, Limbi si literaturi strŏ̌ine, II/ 1981.

C.I. Gulian, « Pascal, si existentialismul », in Revista de filosofie, 15/ 1968.

C.I. Gulian, « Pascal », in Introducere în istoria filosofei moderne, Bucuresti, Editura Enciclopedică Românŭ, 1974.

Silvian Iosifescu, « Mizerie si mŏretie », in Proză si luciditate, Bucuresti, Editura Eminescu, 1974.

Horia Lazŏrr, « Ordinile pascaliene ale realitŏtii », in Revista de filosofie, XXXV (2/ 1988).

Nicolae Maris, « Comunitatea oamenilor de stiintă în optiunea lui Blaise Pascal », in Studii de istorie a filosofiei universale, VI, Bucuresti, Editura Academiei, 1979.

Nicolae Maris, « Blaise Pascal », in Istoria filosofiei moderne si contemporane, vol. I, Bucuresti, Editura Academiei, 1984.

\section{INDEX}

Mots-clés : Pascal, études

Keywords : Pascal, studies

Index géographique : Roumanie

\section{AUTEUR}

HORIA LAZAR

Université de Cluj 